\title{
An Analysis of Structural and Optical Properties Undoped ZnS and Doped (with Mn, Ni) ZnS Nano Particles
}

\author{
Apurba Kr. Das ${ }^{1 *}$, Apurba Kr. Buzarbaruah ${ }^{2}$, Santanu Bardaloi ${ }^{3}$ \\ ${ }^{1}$ Arya Vidyapeeth College, Guwahati, India \\ ${ }^{2}$ Dimoria College, Khetri, India \\ ${ }^{3}$ Gauhati University, Guwahati, India \\ Email: *akdas09@yahoo.com
}

Received January 2, 2013; revised February 4, 2013; accepted March 1, 2013

Copyright (C) 2013 Apurba Kr. Das et al. This is an open access article distributed under the Creative Commons Attribution License, which permits unrestricted use, distribution, and reproduction in any medium, provided the original work is properly cited.

\begin{abstract}
The behavior of nano-particles finds a wide application in opto-electronic and semi-conductor devices. ZnS nano-crystals were grown into poly-vinyl alcohol matrix by chemical route at different weight percentage. Optical properties of both un-doped and doped with $\mathrm{ZnS}$ nano-crystalline compounds were studied. The nano structure was characterized with the help of X-ray diffraction (XRD) and Hi-resolution Transmission Electron Microscopy (HRTEM). Surface morphology was studied with the help of Scanning Electron Microscopy (SEM). The average particle sizes of ZnS, $\mathrm{ZnS}-\mathrm{Ni}$ and $\mathrm{ZnS}-\mathrm{Mn}$ were found to be 6.51, 7.3 and $12 \mathrm{~nm}$ respectively in TEM and that obtained from Debye-Scherrer formula is about 2.3 and $2.5 \mathrm{~nm}$ for undoped and doped $\mathrm{ZnS}$ respectively. Peak of Photo-luminescence (PL) emission spectra was obtained at $375 \mathrm{~nm}$ at room temperature and another peak at $433 \mathrm{~nm}$ for Ni. Again the peak of Photo-luminescence (PL) emission spectra was obtained at $334 \mathrm{~nm}$ at room temperature for $\mathrm{Mn}$, Mn dependant emission was found at $580 \mathrm{~nm}$. These data showed successful doping. PL studies also confirmed presence of dopant in the nano crystallites. Optical absorption studies were carried out with UV-VIS Spectrophotometer and showed a strong absorbance at wavelength $400 \mathrm{~nm}$ with a tendency towards blue shift. Selected area electron diffraction (SAED) shows a set of four well defined rings corresponding to diffraction from different planes of the nano crystallites. HRTEM image showed a well crystalline ZnS doped with $\mathrm{Ni}$ and Mn. Both Raman spectra and XRD studies confirmed the well crystalline states of $\mathrm{ZnS}$.
\end{abstract}

Keywords: Nano-Particle; PVA; XRD; SEM; TEM; HRTEM; SAED

\section{Introduction}

The physical and chemical properties of nano materials like optical absorbance, fluorescence, catalytic activity, electrical and thermal conductivity etc. are significantly different from the corresponding bulk materials. The special properties of nano particles are due quantum size confinement in nano structure and extremely large surface to volume ratio relative to bulk materials and therefore it is possible to keep high percentage of atoms or molecules in lower reactive boundaries.

The synthesis and characterization of nano crystals grown with different chemicals have created interest among the researchers. Low-dimensional semiconductors, like $\mathrm{ZnS}$ composite nano-structured have attracted much interest because of their valuable photoluminescence properties [1]. Chemical growth process is a very simple,

${ }^{*}$ Corresponding author. economical, efficient, and convenient method among the various researchers. In optical sensors, electroluminescence devices, digital displays, etc. doped $\mathrm{ZnS}$ nano materials are being used extensively. Photo luminescent properties and efficiency of $\mathrm{ZnS}$ depend on intrinsic surface states of the particles, and nature of the chemicals treatment employed in their fabrication. Research is also noticed on the application of these types of films in light-emitting materials as well as on their optical properties [2]. The optical light emission in blue-red spectral region is characterized by blue shift at smaller crystallite dimension. We are trying to characterize the properties of $\mathrm{ZnS}$ with different doping agents ( $\mathrm{Ni}$ and $\mathrm{Mn}$ ) with the help of instrument like X-ray Powder Diffractometer (XRD), Scanning Electron Microscopes (SEM), High Resolution Transmission Electron Microscopes (HRTEM), Photo-luminescence spectrometer (PL), UV visible spectrophotometer (UV-VIS), etc. 
Experimental The synthesisation $\mathrm{ZnS}$ nano-particles were done by using Polyvinyl Alcohol (PVA) as a matrix. Different weight $\%$ solutions of PVA and $\mathrm{ZnCl}_{2}$ in deionized water were taken and stirred at $200 \mathrm{rpm}$ in a magnetic stirrer at temperature $70^{\circ} \mathrm{C}$ for 3 hours. The solution was kept overnight for complete dissolution and found to be transparent. A 2 weight $\% \mathrm{Na}_{2} \mathrm{~S}$ solution was added till the whole solution appears milky. The solution was kept over night inside a dark chamber. As soon as the nanostructure formed, it embedded into the gap. The chemical reaction took place as follows

$$
\mathrm{ZnCl}_{2}+\mathrm{Na}_{2} \mathrm{~S}=\mathrm{ZnS}+2 \mathrm{NaCl}
$$

To make different $\%$ of $\mathrm{ZnS}$ :Ni solution, $\mathrm{NiCl}_{2} \cdot 6 \mathrm{H}_{2} \mathrm{O}$ was mixed by weight $\%$ with deionized water. Solution so obtained was mixed with another solution of PVA and $\mathrm{ZnCl}_{2}$. Then the solution was stirred at $200 \mathrm{rpm}$ in a magnetic stirred at constant temperature $70^{\circ} \mathrm{C} .0 .08 \mathrm{M}$ weight $\% \mathrm{Na}_{2} \mathrm{~S}$ solution was added to the solution. Precipitation found was washed with deionized water and taken for study.

To make different $\%$ of $\mathrm{ZnS}: \mathrm{Mn}$ solution, $\mathrm{MnCl}_{2} \cdot 4 \mathrm{H}_{2} \mathrm{O}$ was mixed by weight $\%$ with deionized water. Solution so obtained was mixed with another solution of PVA and $\mathrm{ZnCl}_{2}$. Then the solution was stirred at $200 \mathrm{rpm}$ in a magnetic stirrer at constant temperature $70^{\circ} \mathrm{C} .0 .08 \mathrm{M}$ weight $\% \mathrm{Na}_{2} \mathrm{~S}$ solution was added to the solution. Precipitation found was washed with deionized water and taken for study.

\section{Microstructure Studies}

\subsection{XRD Studies}

The XRD studies shown in Figures 1(a) and (b) were obtained from powder. Diffractogram was obtained from a Philips X'pert Pro Powder diffractometer using $\mathrm{Cu} \mathrm{K} \alpha$ radiation with the operating voltage $40 \mathrm{kV}$ and current 20 $\mathrm{mA}$. The pattern observed was found to be within the nano range $[3,4]$. XRD patterns revealed the films to be polycrystalline [5]. Planes (111), (220) and (311) were found to present which tallied well with the JCPDS card No. 05-0566. The average particle size corresponding to the FWHM was calculated with the Scherrer formulae

$$
D p=\frac{0.94 \lambda}{\beta_{1 / 2} \cos \theta}
$$

and found to be $2.3 \mathrm{~nm}[6]$.

\subsection{Electron Diffraction Studies}

Selected area electron diffraction (SAED) was done with the help of HRTEM. Photo of SAED of undoped ZnS (Figures 2(a)-(c)) also showed a set of three well defined rings corresponding to the planes (111), (220) and (311) in case of undoped $\mathrm{ZnS}$, which is also in good agree-

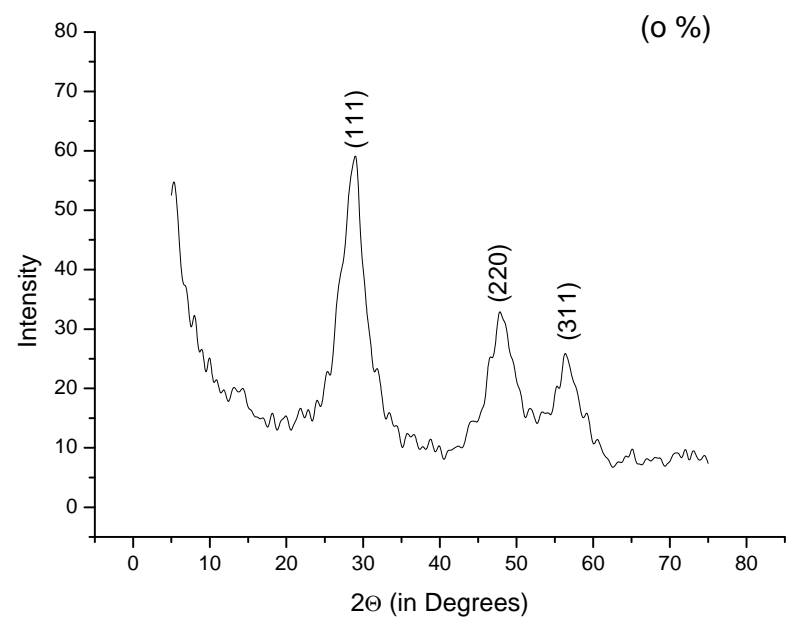

(a)

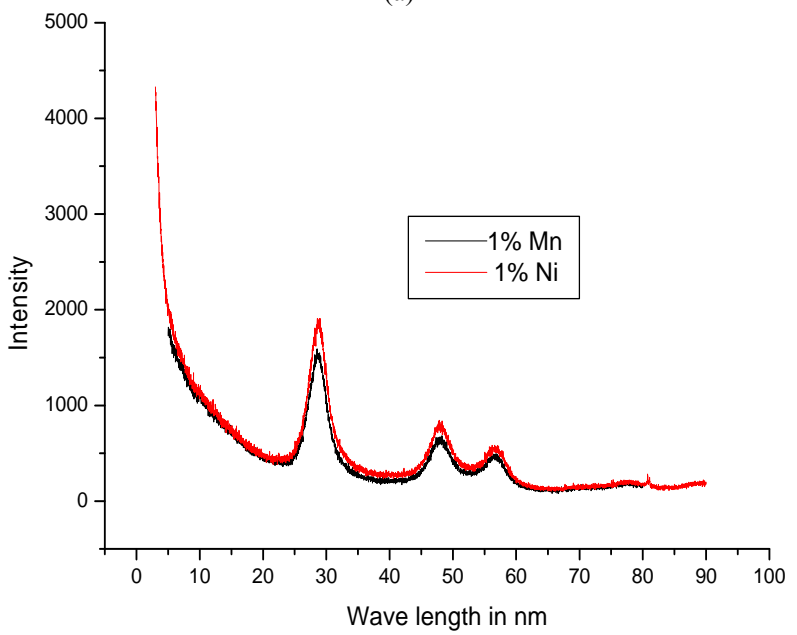

(b)

Figure 1. (a) XRD diffractogram of ZnS; (b) XRD diffractogram of ZnS-Mn.

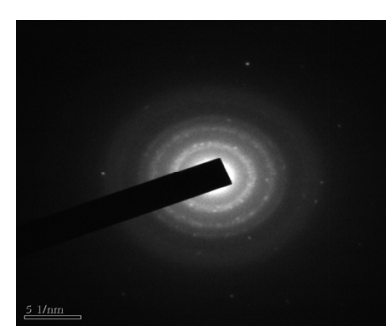

(a)

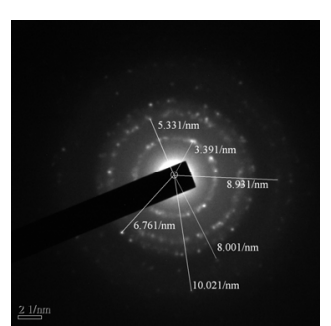

(b)

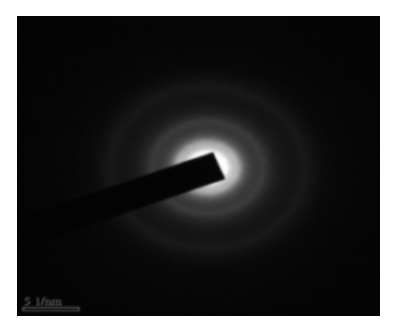

(c)

Figure 2. (a) SAED of ZnS; (b) SAED of ZnS-Ni; (c) SAED of ZnS-Mn. 
ment with that of XRD data.

\subsection{SEM Studies}

Photographs of the nano-crystalline thin film were taken with (JEOL, JSM-6360) SEM and shown in Figure 3. The surface morphology of the film prepared at $70^{\circ} \mathrm{C}$ with PVA was observed and found that all the particles formed not exactly spherical. Study showed that the surface of the film was smooth, uniform and without any crack. The particle sizes of $\mathrm{ZnS}-\mathrm{Ni}, \mathrm{ZnS}-\mathrm{Mn}$ were found to be $7-16$ and $7-15 \mathrm{~nm}$ respectively.

\subsection{Photo Luminescence Studies}

The photo luminescence studies of nano crystalline doped $\&$ un-doped (Figure 4) were done at room temperature by using F-2500 FL Spectrophotometer. In all the measurement the excitation wavelength was $240 \mathrm{~nm}$. Emission spectra showed a broad peak at $378 \mathrm{~nm}$ and another small broad peak at $451 \mathrm{~nm}$ for $\mathrm{ZnS}$ while $375 \mathrm{~nm}$ and $433 \mathrm{~nm}$ for ZnZ-Ni $(0.5 \%)$ and at $382 \mathrm{~nm}$ for ZnS-Ni. For $\mathrm{Mn}$ the emission spectra showed a broad peak at 346 $\mathrm{nm}$ and another small broad peak at $468 \mathrm{~nm}$. PL in this region is due to the presence of $\mathrm{S}$ vacancies in the lattice. PL spectra of $\mathrm{ZnS}: \mathrm{Mn}$ thin film revealed yellow-orange emissions. Mn dependent yellow emission was found at $580 \mathrm{~nm}$ which was also a confirmation successful $\mathrm{Mn}$ doping. Starting with the blue emission (at $468 \mathrm{~nm}$ ), intensity decreases towards the orange emission (at $580 \mathrm{~nm}$ ) [7]. PL spectra of ZnS:Ni revealed yellow-orange emissions.

\subsection{Optical Absorbance Study}

The optical absorbance (Figure 5) of $\mathrm{ZnS}$ was recorded at room temperature using a Double Beam Automated Spectrophotometer (Hitachi-U3210) where the measurement of optical absorbance was done in the range 200 - $800 \mathrm{~nm}$. The wavelength showed strong absorption. The peak of the absorption showed at $287 \mathrm{~nm}$ for $\mathrm{ZnS}$. The peak of the absorption showed blue shift with respect to bulk attributing quantum confinement effect on the nano-particles. Optical absorption studies were carried out with UV-VIS Spectrophotometer and showed a

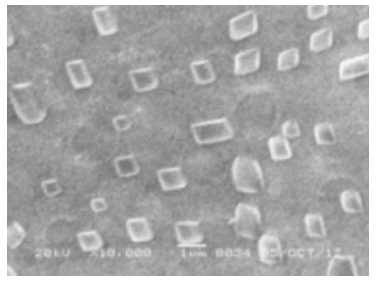

(a)

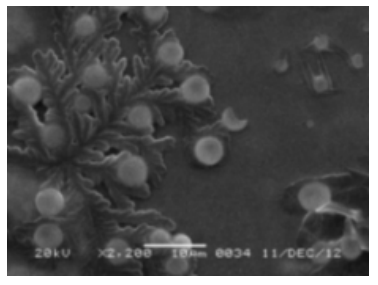

(b)
Figure 3. (a) SEM micrograph of ZnS-Ni; (b) SEM micrograph of ZnS-Mn.

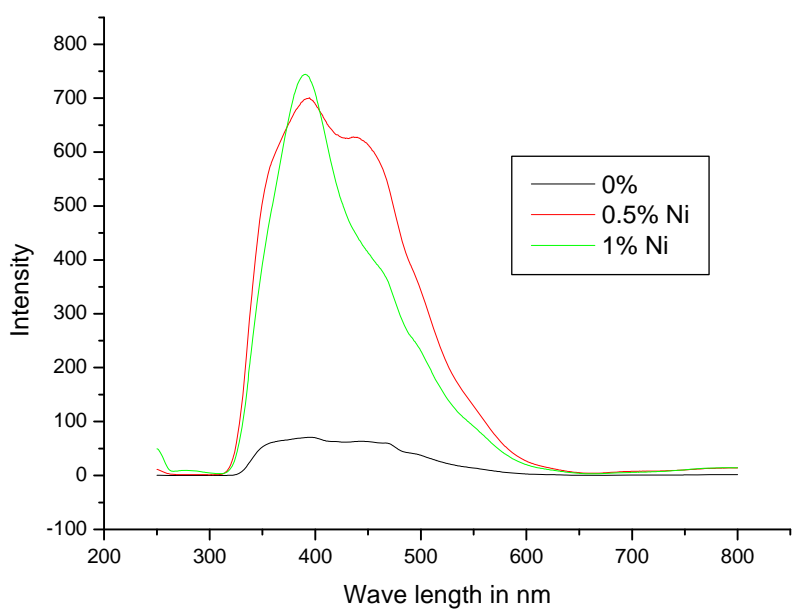

(a)

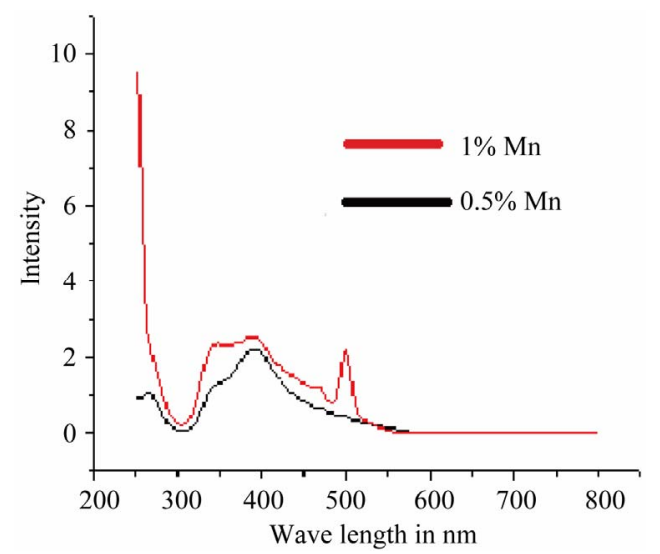

(b)

Figure 4. (a) PL spectra of Zn-S, ZnS-Ni; (b) PL spectra of Zn-S, ZnS-Mn.

strong absorbance at wavelength $400 \mathrm{~nm}$ with a tendency towards blue shift.

Optical absorbance spectrum of $\mathrm{ZnS}-\mathrm{Ni}(0.5 \%, 1.0 \%)$ features a strong peak around $400 \mathrm{~nm}$. This spectra of undoped and Ni doped $\mathrm{ZnS}$ nano crystal are distinguishable. This indicates that Ni doping has effect on the electronic absorption spectra of $\mathrm{ZnS}$ [8]. This may be possible for low doping level.

\subsection{HRTEM}

HRTEM image shows clear lattice fringes of the (001) plane indicating crystal growth along (001) direction. The particle size obtained from HRTEM image is $6 \mathrm{~nm}$ and $7.3 \mathrm{~nm}$ for $\mathrm{ZnS}-\mathrm{Ni}$ and $\mathrm{ZnS}-\mathrm{Mn}$ respectively.

\subsection{Raman Studies}

In Figure 6 the sharp peak of Raman spectra indicated $\mathrm{ZnS}$ in well crystalline state. The peaks at $58 \mathrm{~cm}^{-1}$ and $335 \mathrm{~cm}^{-1}, 347 \mathrm{~cm}^{-1}$ are the characteristic Raman scattering of $\mathrm{ZnS}$ and indexed to LO phonon mode [9]. Another 


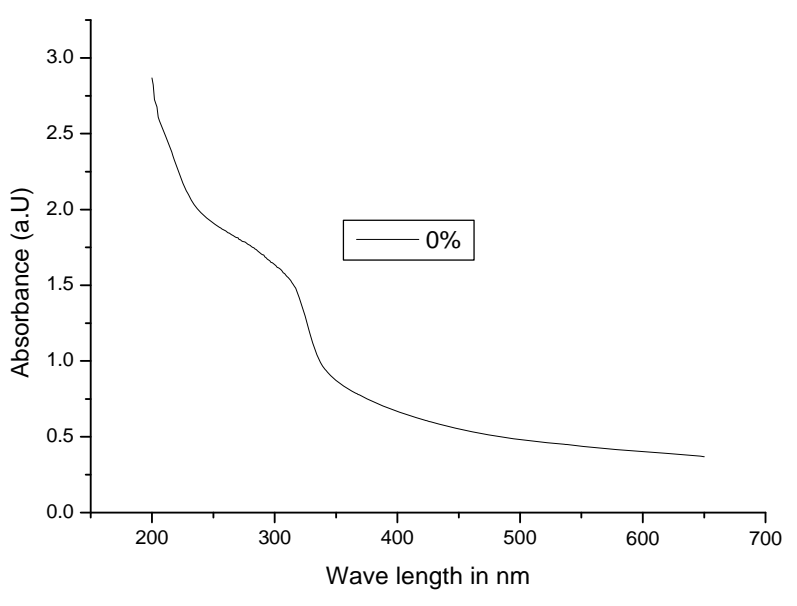

(a)

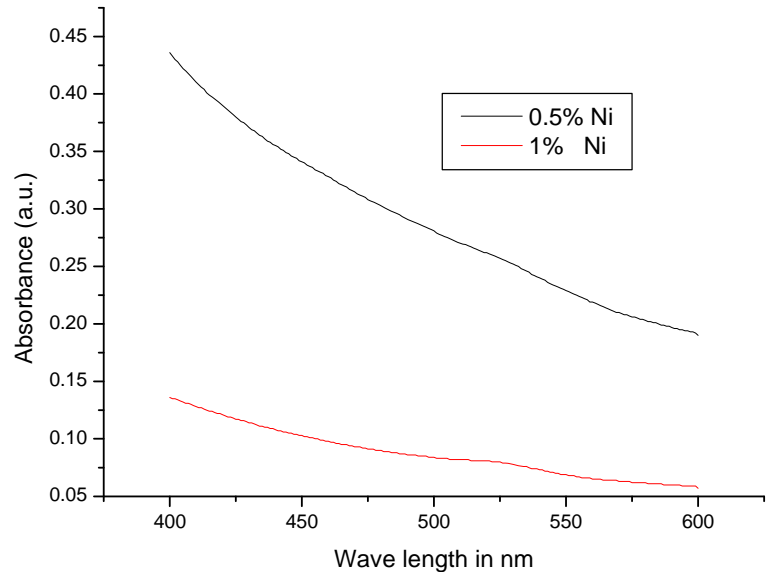

(b)

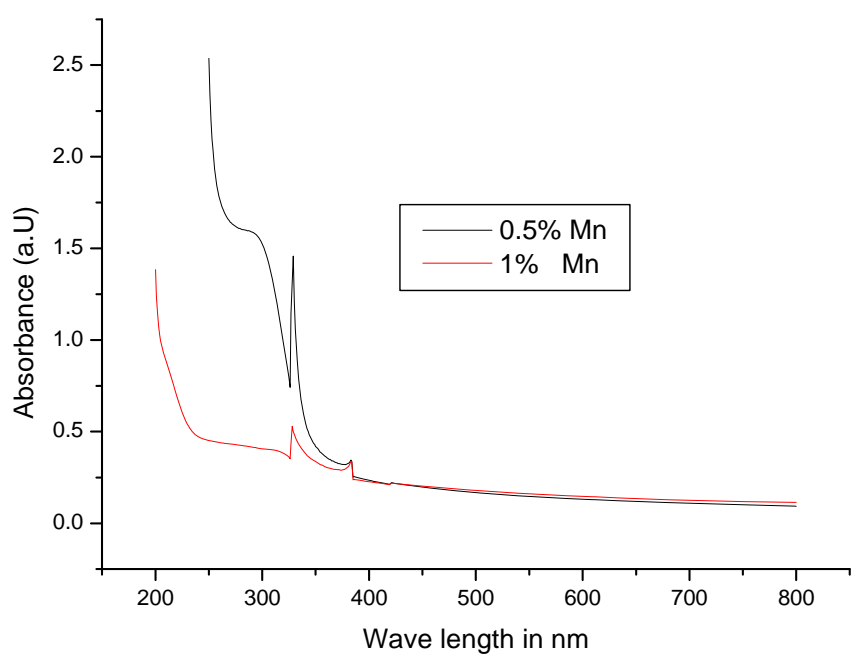

(c)

Figure 5. (a) Absorbance spectra of ZnS; (b) Absorbance spectra of ZnS-Ni; (c) Absorbance spectra of ZnS-Mn.

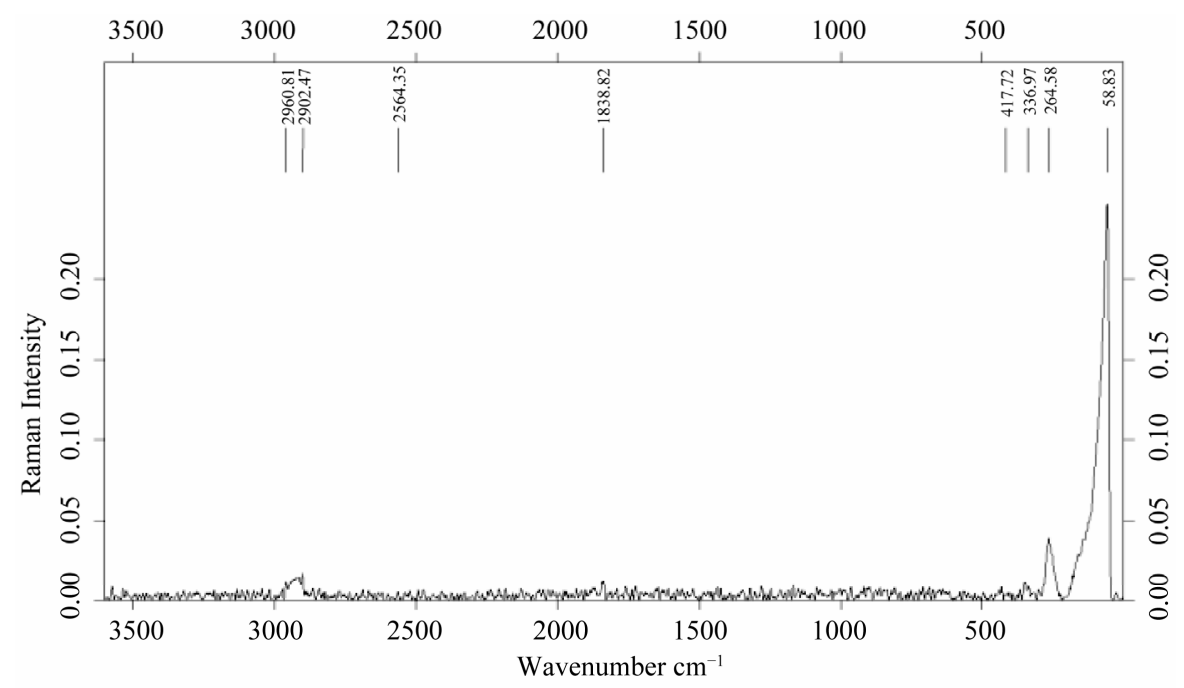

Sample Name: AKD1R

Figure 6. Raman spectra. 
peak at $264 \mathrm{~cm}^{-1}$ corresponds to TO phonon mode [10] of $\mathrm{ZnS}$.

\section{Conclusion}

$\mathrm{ZnS}$ nano crystalline films (un-doped and doped with $\mathrm{Ni}$ and $\mathrm{Mn}$ ) have been synthesized by chemical route. The structural and optical characterization of the films done with the help of XRD, TEM, SEM, SAED, UV-VIS spectrophotometer and PL reveals formation of doped as well as un-doped nano particles. The emission bands at $377 \mathrm{~nm}, 449 \mathrm{~nm}$ (both for 0\%), $375 \mathrm{~nm}, 433 \mathrm{~nm}$ (both for $0.5 \% \mathrm{Ni}$ ) and $382 \mathrm{~nm}$ for $1 \% \mathrm{Ni}$ may be attributed to impurities or defect state. Similarly the emission bands with respect to $375 \mathrm{~nm} \& 451 \mathrm{~nm}$ for ZnS-Mn may be attributed to defect states. The third emission band at 496 $\mathrm{nm}$ may be attributed in the $3 \mathrm{~d}$ shell transition of $\mathrm{Mn}^{2+}$. The sharp peak of Raman spectra indicated $\mathrm{ZnS}$ in well crystalline state which is in good agreement with that obtained from XRD result.

\section{Novelty Statement}

From Photo luminescence study it has been observed that in case of $\mathrm{ZnS}-\mathrm{Ni}$, with the increase of doping\% (from $0.5-1.0)$, the intensity of doping related emission spectra is found to be increased. In case of $\mathrm{ZnS}-\mathrm{Mn}$, with the increase of doping\% (from $0.5-1.0$ ), the intensity found to be increased. It is also observed from the PL that with the increase of doping\% (from 0.5 - 1.0) of both $\mathrm{ZnS}-\mathrm{Ni}$ and $\mathrm{ZnS}-\mathrm{Mn}$, there is blue shift of doping related emission spectra due to quantum confinement effect. The bands with respect to $375 \mathrm{~nm} \& 451 \mathrm{~nm}$ may be attributed to defect states. The sharp peaks of Raman spectra indicated $\mathrm{ZnS}$ nano particles were in well crystalline state.

\section{Acknowledgements}

The authors are very much thankful to Dr. P. K. Baruah, Department of Chemistry, Gauhati University for his help in taking UV-VIS and PL data. We are also thankful to SAIF, Shillong for providing TEM and SEM facility.
Authors are also like to offer their gratitude to SAIF, Guwahati, Department of Instrumentation \& USIC, Gauhati University for providing XRD \& XRF facility. The authors are also like to offer their heart felt gratitude and thanks to CIF and dept. of Nano-Science and Technology, IITG for providing HRTEM and XRD facility. The authors are also grateful to Prof. K. C. Sarma, Head, Dept. of Instrumentation \& USIC, Gauhati University for constant guidance and help.

\section{REFERENCES}

[1] P. Yang, M. Lu, D. Xu, D. Yuan, J. Chang, G. Zhou and M. Pan, Applied Physics A, Vol. 74, pp. 257-259.

[2] T. M. Thi, B. H. Van and P. Van Ben, Journal of Nonlinear Optical Physics \& Materials, Vol. 19, pp. 237-245. doi:10.1142/S021886351000525X

[3] B. S. Rema Devi, R. Raveendran and A. V. Vaidyan, Pramana Indian Academy of Sciences, Vol. 68, 2007, pp. 679-687.

[4] S. Kumar, N. K. Verma and M. L. Singla, Chalcogenide Letters, Vol. 8, 2011, pp. 561-569.

[5] A. Goudarzi, G. M. Aval, S. S. Park, M.-C. Choi, R. Sahraei, M. Habib Ullah, A. Avane and C.-S. Ha, Chemistry of Materials, Vol. 21, 2009, pp. 2375-2385. doi: $10.1021 / \mathrm{cm} 803329 \mathrm{w}$

[6] G. Murugadoss, B. Rajamannan and U. Madhusudhanana, Chalcogenide Letters, Vol. 6, 2009, pp. 197-201.

[7] S. P. Singh, O. J. Perales-Perez, M. S. Tomar and O. V. Mata, Physica Status Solidi (c), Vol. 1, 2004, pp. 811-814. doi:10.1002/pssc.200304292

[8] K. Jayanthi, S. Chawla, H. Chander and D. Haranath, Journal of Nonlinear Optical Physics and Materials, Vol. 42, 2007, p. 976.

[9] C. Lan, K. Q. Hong, W. Z. Wang and G. H. Wang, Solid State Communication Solid State Communications, Vol. 125, 2003, pp. 455-458. doi:10.1016/S0038-1098(02)00914-6

[10] W. G. Nilsen, Physical Review, Vol.182, 1969, pp. 838850. 